\title{
Review of: "Chlorella vulgaris functional alcoholic beverage: Effect on propagation of cortical spreading depression and functional properties"
}

Simeone Chianese ${ }^{1}$

1 Università degli Studi della Campania Luigi Vanvitelli

Potential competing interests: The author(s) declared that no potential competing interests exist.

Comment to the paper: "Chlorella vulgaris functional alcoholic beverage: Effect on propagation of cortical spreading depression and functional properties" by Danielli M. M. Dantas et al.

\section{General comment:}

The manuscript deals with investigations on Chlorella vulgaris functional alcoholic beverage. Investigations were carried out in vitro by radical scavenging tests, and in vivo, by modeling cortical spreading depression in rat brains.

The manuscript is suitable to be published in this journal; however, some points should be addressed before publication.

Some minor language mistakes are present that should anyway be corrected.

\section{Results}

Please, improve comparison between your findings and literature data.

The whole characterization of microalgae should be included.

The whole characterization of the extracted solution should be included.

Please, include statistical analysis results. 